\title{
Review of the EFT treatment of quarkonium at finite temperature
}

\section{Jacopo Ghiglieri*}

McGill University, Department of Physics, 3600 rue University, Montreal QC H3A 2T8, Canada

E-mail: jacopo.ghiglieri@physics.mcgill.ca

Heavy quarkonium is one of the most investigated probes of the medium produced in heavy-ion collisions. In the past few years progress has been made in the description of its in-medium dynamics from QCD. Non-relativistic EFTs in particular allow one to define and compute rigorously the potential governing the real-time evolution of the bound state from QCD, showing that it is composed of a real and an imaginary part. The latter encodes the effect of scattering with the light constituents of the plasma and is oftentimes responsible for the dissociation of the bound state. The EFT approach and its main results, as well as comparisons with recent lattice data and phenomenological applications, are briefly reviewed here.

Xth Quark Confinement and the Hadron Spectrum

8-12 October 2012

TUM Campus Garching, Munich, Germany

\footnotetext{
${ }^{*}$ Speaker.
} 


\section{Introduction}

The phase diagram of QCD in the region of vanishing chemical potential is actively explored in heavy ion collision experiments at RHIC and LHC. Lattice calculations (see [1] for a recent review) predict a crossover transition to a larger number of degrees of freedom, typical of a deconfined medium (the Quark-Gluon Plasma), for temperatures $T$ ranging from 150 to $200 \mathrm{MeV}$. On the experimental side, the characterization of the properties of the medium relies either on its bulk properties, which find an effective description in hydrodynamics, or in hard probes, i.e. energetic particles not in equilibrium with the medium.

Heavy quarkonium has been one of the most actively investigated hard probes for the past 26 years. In 1986 Matsui and Satz [2] hypothesized that colour screening in a deconfined medium would have dissociated the $J / \psi$, resulting in a suppressed yield in the easily accessible dilepton decay channel and yielding a striking QGP signature. Such a suppressed yield has been indeed observed in heavy ion collision experiments at SPS, RHIC and LHC (see [3] for a review), the current understanding being that it cannot be explained by cold nuclear matter effects alone, i.e. those caused by a confined nuclear medium. Furthermore the LHC results have opened up the frontier of the cleaner bottomonium probe with the availability of quality data on the $\Upsilon$ resonances (see [4] for the latest CMS results).

On the theory side, a great deal of the studies of the in-medium dynamics of the $Q \bar{Q}$ bound states has been carried out with phenomenological potential models, first introduced in [5], where all medium effects are encoded in a $T$-dependent potential plugged in a Schrödinger equation. We refer to [6-8] for recent reviews. The derivation of such models from QCD was however not established. Moreover, lattice calculations of free energies and other quantities [9, 10] obtained from correlation functions of Polyakov loops are often taken as input for the $T$-dependent potential. Although these quantities have been thought to be related to the colour-singlet and colour-octet heavy quark potentials at finite temperature $[11,10]$, a precise relation was still lacking in the literature, as pointed out in [12].

In the past few years, a considerable effort has been devoted to the derivation of a proper potential from QCD. In [13] (see also [14]) it was shown that the perturbative real-time potential obtained by analytical continuation of a rectangular Wilson loop shows a screened real part and a large imaginary part (for $T \gg 1 / r \sim m_{D}, m_{D}$ being the Debye mass, the imaginary part is much larger than the real part) which is due to the scattering of the virtual potential gluons with the light particles (quarks and gluons) of the medium, in what is called Landau damping. Further phenomenological studies have shown the numerical importance of this imaginary part [15-17] when solving a Schrödinger equation. A generalization to anisotropic plasmas has been performed in $[18-20]$ and used in $[21,22]$ (coupled with a hydrodynamical evolution) to obtain predictions on the suppression rates.

The first-principle derivation of potentials from QCD was further improved with the development of an Effective Field Theory (EFT) framework [23-31], which will be at the center of this contribution. This approach, started in [23] (see also [24] for the abelian analog), relies on the well-established framework of $T=0$ Non-Relativistic (NR) EFTs of QCD and extends it to finite temperatures, giving to the potential a modern, rigorous definition as a matching coefficient of the EFT that arises when the scale $m v$ is integrated out, where $m$ is the heavy quark $(Q)$ mass and $v \ll 1$ 
is the relative velocity of the constituents of the NR bound state. At zero temperature this EFT is called Potential Non-Relativistic QCD (pNRQCD) [32, 33].

This contribution is structured as follows: in Sec. 2 we will illustrate the basic principles of the EFT framework. In sections 3 and 4 we will apply those principles to the real-time dynamics of bound states in two interesting temperature regimes, $T>m v$ and $T<m v$ respectively. In Sec. 5 we will compare the thermal widths obtained within the EFT framework with other approaches in the literature and show how dissociation cross sections can be extracted from the EFT calculations. In Sec. 6 we will deal with Euclidean quantities and their related thermodynamical free energies, showing how they relate to the $Q \bar{Q}$ potentials. In Sec. 7 we will briefly review some interesting recent developments and finally in Sec. 8 we will draw our conclusions.

\section{EFTs}

In a scenario where a physical system is described by two scales $\Lambda \gg m$, a low-energy EFT describing the physics at the scale $m$ is constructed by integrating out $\Lambda$, i.e. integrating out modes of energy/momentum above a given cut-off $\mu, \Lambda \gg \mu \gg m$, and imposing that the EFT and the theory it is derived from are equivalent below the cut-off. If the system is characterized by more than two scales and if these scales are hierarchically ordered, the procedure can be iterated, creating a tower or hierarchy of EFTs. If two scales are not hierarchically separated, they should then be integrated out in the same step.

This line of action is at the base of the above-mentioned $T=0$ NR EFT framework. As we remarked, the expansion parameter there is $v \ll 1$, giving rise to the hierarchy of scales $m \gg$ $m v \gg m v^{2}$, where $m$ is the heavy quark mass, $m v$ is the typical relative momentum or inverse radius and $m v^{2}$ the kinetic/binding energy. The framework is constructed by integrating out first the mass scale, yielding Non-Relativistic QCD (NRQCD) [34, 35]. Its Lagrangian is organized as an expansion in the inverse mass. The second step is the integration of the scale $m v$, which leads to pNRQCD [32,33]. The relative hierarchical position of $\Lambda_{\mathrm{QCD}}$ and $m v$ establishes whether this integration is to be performed perturbatively or non-perturbatively. The former case leads to weakly-coupled pNRQCD, which we illustrate briefly because the EFTs developed at finite temperature, which will be introduced later, will share its basic form.

The degrees of freedom in the $Q \bar{Q}$ sector are conveniently written as a colour-singlet and a colour-octet $Q \bar{Q}$ bilinear field, which can interact through ultrasoft ( $E, p \sim m v^{2}$ gluons). This cutoff is effectively expressed through a multipole expansion. In detail, the Lagrangian reads $[32,33]$

$$
\begin{gathered}
\mathscr{L}_{\mathrm{pNRQCD}}=-\frac{1}{4} F_{\mu v}^{a} F^{a \mu v}+\sum_{i=1}^{n_{f}} \bar{q}_{i} i D D q_{i}+\int d^{3} r \operatorname{Tr}\left\{\mathrm{S}^{\dagger}\left[i \partial_{0}-h_{s}\right] \mathrm{S}+\mathrm{O}^{\dagger}\left[i D_{0}-h_{o}\right] \mathrm{O}\right. \\
\left.+V_{A}\left(\mathrm{O}^{\dagger} \mathbf{r} \cdot g \mathbf{E} \mathrm{S}+\text { H.c. }\right)+\frac{V_{B}}{2} \mathrm{O}^{\dagger}\{\mathbf{r} \cdot g \mathbf{E}, \mathrm{O}\}+\ldots\right\} .
\end{gathered}
$$

The fields $\mathrm{S}=S \mathbf{1}_{c} / \sqrt{N_{c}}$ and $\mathrm{O}=O^{a} T^{a} / \sqrt{T_{F}}$ are the $Q \bar{Q}$ colour-singlet and colour-octet fields respectively, $q_{i}$ are the light quarks, in $n_{f}$ flavours, $T_{F}=1 / 2, \mathbf{E}$ is the chromoelectric field, $i D_{0} \mathrm{O}=$ $i \partial_{0} \mathrm{O}-g A_{0} \mathrm{O}+\mathrm{O} g A_{0}$ and H.c. stands for Hermitian conjugate. The trace is over colour and spin indices. The dots in the last line stand for higher-order terms in $r$ and $1 / m$. 
The dependence on the scales $m$ and $m v \sim m \alpha_{\mathrm{S}}$ is encoded in the Wilson coefficients; $V_{A}$ and $V_{B}$ are at leading order $V_{A}=V_{B}=1$, whereas the singlet and octet Hamiltonians have the form $\left(\mathbf{p} \equiv-i \nabla_{\mathbf{r}}\right)$

$$
h_{s}=\frac{\mathbf{p}^{2}}{m}-C_{F} \frac{\alpha_{\mathrm{s}}}{r}+\ldots, \quad h_{o}=\frac{\mathbf{p}^{2}}{m}+\frac{1}{2 N_{c}} \frac{\alpha_{\mathrm{s}}}{r}+\ldots
$$

The dots in these equations stand for higher orders in the $1 / m$ and $\alpha_{\mathrm{s}}$ expansions, such as spinand momentum-dependent terms or radiative corrections to the Coulomb potentials. $N_{c}=3$ is the number of colours and $C_{F}=4 / 3$ in QCD. In the power-counting of the EFT the explicitly-shown terms are of the same size and give rise to a Coulombic bound state. At the zeroth order in the multipole expansion the equation of motion for the singlet field arising from Eq. (2.1) is then a simple Schrödinger equation, resulting in a spectrum made by the (QCD) Bohr levels $E_{n}=-4 m \alpha_{\mathrm{s}}^{2} /\left(9 n^{2}\right)$, whereas the octet potential is repulsive and does not support bound states but a continuum of scattering states. Higher orders in the multipole expansion, such as the chromoelectric dipole couplings on the second line of Eq. (2.1), are responsibile for retardation effects such as those yielding the Lamb shift in hydrogen spectroscopy.

At finite temperature one also encounters the scales that characterize the thermal medium: its temperature $T$, the electric (Debye) screening mass $m_{D} \sim g T$ and a magnetic mass of order $g^{2} T{ }^{1}$ In the weak-coupling scenario that has so far been investigated in the EFT approach, these scales develop a hierarchy. In order to construct a finite temperature EFT framework analogous to the one just illustrated, one needs then to put these scales together with the NR ones in a global hierarchy. Many such hierarchies are possible and phenomenologically sensible. The latter requirement translates into $T \ll m$.

Once a hierarchy is fixed, one proceeds to integrate out scales along the procedure sketched above. Since $m \gg T$, the first step is always the integration of the mass, leading again to NRQCD. Since an EFT and its matching coefficients are independent of the hierarchy of scales below the cutoff, its Lagrangian is identical to the zero-temperature case.

After this first step, the subsequent ones differ in the different cases, which in the following we will group into two regions: the one where $T \gg m v$ and the one where $T \ll m v$, which can be of relevance for the ground state of bottomonium. The case $T \sim m v$ has been dealt with in QED in [27]; its QCD investigation is underway in [36]. When integrating out the thermal scales, existing finite-T EFTs of QCD such as the Hard Thermal Loop (HTL) effective theory [37] are employed.

In all cases, once the scale $m v$ is integrated out, the resulting Lagrangian is similar to the one of pNRQCD, with colour-singlet and colour-octet fields and a Schrödinger equation picture appearing as the zeroth-order equation of motion. The potential is then rigorously defined as a matching coefficient; all scales above $m v^{2}$ will contribute to it.

The different possibilities for the hierarchies and the EFTs that arise out of them are pictured in Fig. 1. We conclude this Section by noting that the NR EFT framework inherits all the strong points of EFTs. In particular, it has a well-defined power counting, which allows for easy estimations of the size of possible contributions to interesting observables, and it is systematically improvable,

\footnotetext{
${ }^{1}$ We do not distinguish between $T$ and $\pi T$ or multiples thereof in the text. For what concerns the magnetic mass, we never reach an accuracy where its contribution becomes relevant, so we need not worry about its non-perturbative treatment.
} 

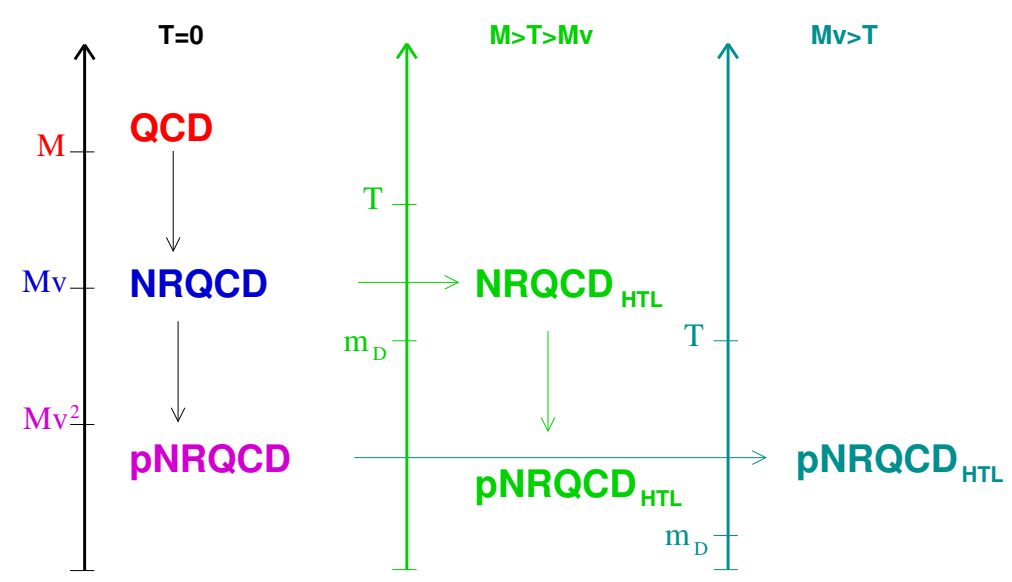

Figure 1: Possible hierarchies and the EFTs they generate. Figure taken from [38].

i.e. new operators and matching coefficient can be added to the Lagrangian and computed in order to reach the desired accuracy in the calculation of the above-mentioned observables.

\section{The $m \gg T \gg m v$ region}

In this region the temperature scale is to be integrated first, obtaining an EFT of NRQCD called NRQCD ${ }_{\text {HTL }}[39,40]$, where the gauge and light-quark sector is described by the HTL Lagrangian. The second step depends on the existence of a hierarchical separation between $m_{D}$ and $m v$. If $m_{D} \sim m v$ the two scales are to be integrated out at the same time. If, on the other hand, $m v \gg m_{D}$, one needs two separate steps. For reasons that will become clearer later, the case $m v \ll m_{D}$ is not relevant.

In the first case one obtains a pNRQCD-like EFT named pNRQCD $D_{\mathrm{HTL}}$, where the potential, obtained by matching from NRQCD $\mathrm{HTL}_{\mathrm{H}}$ in the real-time formalism of Thermal Field Theory, reproduces the earlier result of [13], thus bringing it in a consistent EFT picture [23]. This derivation furthermore shows the computational advantage of the real-time formalism over the imaginary-time one for this class of Minkowskian observables. The colour-singlet potential reads

$$
\left.V_{s}(r)\right|_{T \gg m v \sim m_{D}}=-C_{F} \frac{\alpha_{\mathrm{s}}}{r} e^{-m_{D} r}-C_{F} \alpha_{\mathrm{s}} m_{D}+i 2 C_{F} \alpha_{\mathrm{s}} T \int_{0}^{\infty} d t\left(\frac{\sin \left(m_{D} r t\right)}{m_{D} r t}-1\right) \frac{t}{\left(t^{2}+1\right)^{2}},
$$

where $m_{D}^{2}=g^{2} T^{2}\left(1+n_{f} / 6\right)$. We remark that the real part scales like $\alpha_{\mathrm{s}} m_{D}$ (recall that $m v \sim$ $\left.1 / r \sim m_{D}\right)$. The imaginary part is Bose-enhanced and scales like $\alpha_{\mathrm{s}} T$; it is thus much larger, as we mentioned before. Hence, one can expect bound states to be dissociated in this regime.

If one moves to the second possibility $m v \gg m_{D}$, the integration procedure has to go through an intermediate step. The real part is dominated by the $T=0$ Coulomb term, whereas the imaginary part at the scale $m v$ is IR divergent and is regulated in dimensional regularization. Once the UVdivergent contribution of the smaller scale $m_{D}$ is added up, the following finite result arises [23]

$$
\left.V_{s}(r)\right|_{T \gg m v \gg m_{D}}=-C_{F} \frac{\alpha_{\mathrm{s}}}{r}-\frac{C_{F}}{2} \alpha_{\mathrm{s}} r m_{D}^{2}+i \frac{C_{F}}{6} \alpha_{\mathrm{s}} r^{2} T m_{D}^{2}\left(\ln \left(r m_{D}\right)^{2}+2 \gamma_{E}-\frac{8}{3}\right)
$$


where $\gamma_{E}$ is the Euler-Mascheroni constant. As pointed out in [41] (see also [24] for QED), the real and imaginary parts of this potential become parametrically of the same size when $T \sim m \alpha_{\mathrm{s}}^{2 / 3} .{ }^{2}$ This then constitutes a parametric estimate of the dissociation temperature, where the dissociation criterion popularized by potential models, i.e. disappearance of the binding energy, is replaced by the equality of binding energy and thermal width. A numerical estimate has been performed in [27] for the $\Upsilon(1 S)$ and is reported in Table 1.

\begin{tabular}{|c|c|}
\hline$m_{c}(\mathrm{MeV})$ & $T_{d}(\mathrm{MeV})$ \\
\hline$\infty$ & 480 \\
5000 & 480 \\
2500 & 460 \\
1200 & 440 \\
0 & 420 \\
\hline
\end{tabular}

Table 1: Dissociation temperature for the $\Upsilon(1 S)$ for different values of the charm mass. Table and results are taken from [27].

\section{The $m v \gg T \gg m v^{2}$ region}

In this region the thermal medium acts as a perturbation to a Coulombic bound state, but it still modifies the potential. Furthermore, as argued in [42], hierarchies where $m v>T>m v^{2}$ could be of relevance for the ground states of bottomonium, where $m v \sim m \alpha_{\mathrm{s}} \sim 1.5 \mathrm{GeV}$ and $T$ or even $\pi T$ are smaller in current experiments.

In this region one starts by integrating out $m v$ from NRQCD, obtaining standard PNRQCD. The next step, the integration of the temperature, leads to a different version of $\mathrm{pNRQCD} \mathrm{HTL}_{\mathrm{HT}}$ than the one considered above. Its Lagrangian has been written and some of its matching coefficients have been computed in [25, 29].

Further steps depend on the hierarchy (if any) between $m v^{2}$ and $m_{D}$. In [25] the case $m v^{2} \gg m_{D}$ has been explored in detail, leading to a calculation of the thermal modifications to the spectrum and of the thermal width up to order $m \alpha_{\mathrm{s}}^{5}$ in the power-counting of the EFT. Before we briefly illustrate these results, we remark that other hierarchical possibilities have been considered in the static case in [23] and in QED in [27].

In order to obtain the spectrum and width to order $m \alpha_{s}^{5}$, as well as to compute the matching coefficients of pNRQCD $\mathrm{HTL}_{\mathrm{HL}}$, one has to compute loop diagrams in pNRQCD with loop momentum of order $T$ or in pNRQCD ${ }_{\text {HTL }}$ with loop momenta of order $m v^{2}$ or $m_{D}$. These loop diagrams arise from the dipole interaction vertices in Eq. (2.1); the relevant diagrams for the desired accuracy are shown in Fig. 2. As in the previous section, we encounter intermediate UV or IR divergences which we regulate in dimensional regularization. The potential, which in the EFT framework is not an observable, shows IR divergences. The spectrum and the width, being instead observables, are of course finite, the divergences cancelling in the sum of the contributions from different scales.

\footnotetext{
${ }^{2}$ The Coulombic real part implies a Coulombic power counting, with $m v \sim 1 / r \sim m \alpha_{\mathrm{s}}$. Logarithms of the coupling are not considered in this estimate
} 


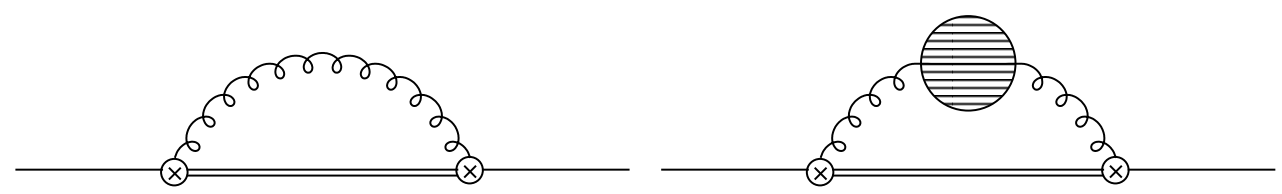

Figure 2: Loop diagrams in $\mathrm{pNRQCD}$ and $\mathrm{pNRQCD}_{\mathrm{HTL}}$ contributing to our calculation. Single lines are colour-singlet $Q \bar{Q}$ states, double lines are colour octets, curly lines are gluons, vertices are chromoelectric dipoles and the blob is the gluon self-energy. The imaginary part of the first diagram yields the singlet-tooctet decay mechanism, whereas the second one gives the Landau damping contribution to the width.

The shift to the binding energy induced by the thermal medium, which translates into a mass shift for the bound state, reads to order $m \alpha_{\mathrm{s}}^{5}$

$$
\begin{aligned}
& \left.\delta E_{1 S}^{\text {(thermal) }}\right|_{m v \gg T \gg m v^{2}}=\frac{34}{27} \pi \alpha_{\mathrm{s}}^{2} T^{2} a_{0} \\
& +\frac{E_{1} \alpha_{\mathrm{s}}^{3}}{\pi}\left[\frac{7225}{324}\left(\log \frac{4 \pi^{2} T^{2}}{E_{1}^{2}}-2 \gamma_{E}\right)+\frac{128}{81} L_{1 S}\right] \\
& +3 a_{0}^{2} \alpha_{\mathrm{s}} T\left\{\frac{8}{3} \zeta(3) \alpha_{\mathrm{s}} T^{2}-\left[\frac{3}{2 \pi} \zeta(3)+\frac{\pi}{3}\right] \frac{4}{3} m_{D}^{2}\right\},
\end{aligned}
$$

where $a_{0}=3 /\left(2 m \alpha_{\mathrm{s}}\right)$ is the Bohr radius and $L_{1 S}$ is the QCD equivalent of the Bethe log in the hydrogen spectrum. We refer to $[43,44]$ for its definition and evaluation, which yields $L_{1 S}=$ -81.5379 .

The thermal width reads instead

$$
\begin{aligned}
\left.\Gamma_{1 S}^{(\text {thermal })}\right|_{m v \gg T \gg m v^{2}}= & \frac{1156}{81} \alpha_{\mathrm{s}}^{3} T+\frac{7225}{162} E_{1} \alpha_{\mathrm{s}}^{3} \\
& -\frac{4}{3} a_{0}^{2} \alpha_{\mathrm{s}} T\left[m_{D}^{2}\left(\ln \frac{E_{1}^{2}}{T^{2}}+2 \gamma_{E}-3-\log 4-2 \frac{\zeta^{\prime}(2)}{\zeta(2)}\right)+8 \pi \ln 2 \alpha_{\mathrm{s}} T^{2}\right] \\
& +\frac{32}{9} \alpha_{\mathrm{s}} T m_{D}^{2} a_{0}^{2} I_{1 S}
\end{aligned}
$$

where $I_{1 S}=-0.49673$ is another Bethe logarithm. We refer to [25] for details. The terms on the first line of this equation arise from the imaginary part of the diagram on the left of Fig. 2 and correspond to the absorption of a thermal gluon by the bound state and its subsequent decay into a colour-octet state, a process we call singlet-to-octet decay. The terms on the second and third lines, on the other hand, arise from the imaginary part of the second diagram and are instead due to scattering of the virtual gluon with the light constituents, i.e. to Landau damping. In the power counting of $\mathrm{pNRQCD} \mathrm{HTL}_{\mathrm{HT}}$ singlet-to-octet decay is dominant over Landau damping as long as $m v^{2}>m_{D}$, as in this case. We will return to these processes in the next section.

We conclude this section by reporting on the findings of [45, 46] (see also [47]). There, the authors employed the lattice formulation of NRQCD to access $b \bar{b}$ bound states on the lattice at finite temperature. In [46] they used Bayesian MEM techniques to reconstruct the spectral function and thus extract $\delta E$ and $\Gamma$ as a function of the temperature. These results are shown in Fig. 3. The authors then proceeded to compare their results with the leading term of the EFT results, represented by the first term on the r.h.s. of Eqs. (4.1) and (4.2). They found them to be compatible for $m_{b}=5 \mathrm{GeV}$ and $\alpha_{\mathrm{s}} \approx 0.4$. 

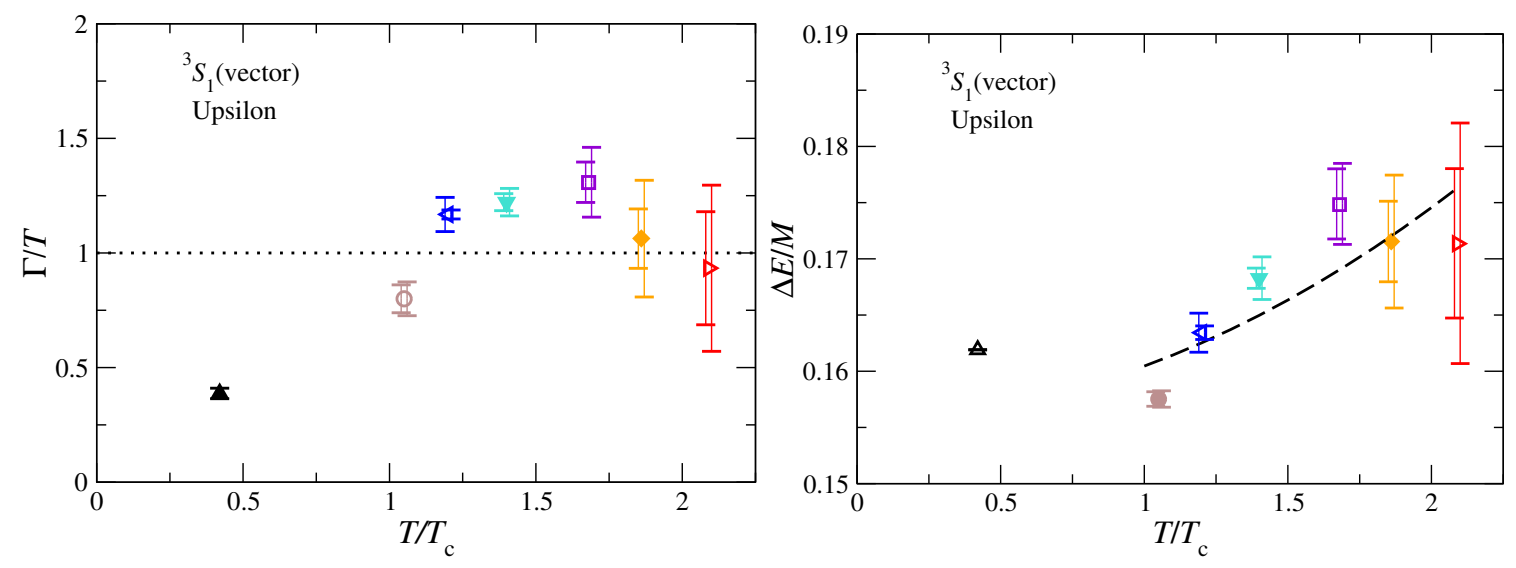

Figure 3: Plots of $\Gamma$ and $\delta E$ in the vector $(\Upsilon)$ channel from the lattice calculation of [46]. Figures taken from that reference. The dashed line on the right is the leading term of our EFT calculation [25], $\frac{34}{27} \pi \alpha_{\mathrm{s}}^{2} T^{2} a_{0}$, for $m_{b}=5 \mathrm{GeV}$ and $\alpha_{\mathrm{s}}=0.4$.

\section{The thermal width in the EFT and in the literature}

In the previous sections we have identified two processes that are responsible for the appearance of the thermal width: these are the singlet-to-octet decay $\left(g+\Psi \rightarrow(Q \bar{Q})_{8}\right.$, where $\Psi$ is the bound state and $(Q \bar{Q})_{8}$ the $Q \bar{Q}$ pair in the colour-octet state) and Landau damping $(p+\Psi \rightarrow$ $p+(Q \bar{Q})_{8}$, where $p=g, q, \bar{q}$ is a light parton). Analogous processes had been previously considered in the literature, going under the names of gluo-dissociation [48, 49] and quasi-free dissociation [50,51] (see [52-54] for recent applications) respectively. In these approaches a $T=0$ cross section for the process at hand, sometimes complemented by momentum-independent thermal masses, was convoluted over a thermal distribution for the incoming parton, i.e.

$$
\Gamma=\int_{q_{\min }} \frac{d^{3} q}{(2 \pi)^{3}} f_{p}(q) \sigma(q) v_{\mathrm{rel}}
$$

where $f_{p}$ is either the Fermi-Dirac or Bose-Einstein distribution, $q$ is the incoming momentum, with kinematic minimum for dissociation $q_{\min }$, and $v_{\text {rel }}$ is the relative velocity. Cross sections employed in the literature have been the Bhanot-Peskin one [55] for gluo-dissociation and [56] or more recently $[57,58]$ for quasi-free dissociation.

In [30, 36] (see also [59]) we have undertaken a comparison between the EFT results and these phenomenological approaches. For what concerns gluo-dissociation, in [30] we have shown that our results (such as the first line of Eq. (4.2)) correspond to the formulation of Eq. (5.1) and that the Bhanot-Peskin cross section of [55] represents a large- $N_{c}$ limit of the one that can be extracted from the EFT framework, which in turn correctly incorporates the effect of the repulsive octet potential on the final state. An equivalent cross section has also been derived in [60] (see also [61, 62]). The EFT approach and its power counting clearly constrain the validity region of the gluo-dissociation approach to temperatures smaller than $m v$, so that the dipole approximation employed is valid, and larger than $m v^{2}$, the threshold for dissociation. If the screening mass becomes of the same order or larger than this latter scale, Hard Thermal Loop resummation becomes necessary and the cross section is no longer temperature-independent. 
For what concerns quasi-free dissociation, our analysis [36, 59] shows that Eq. (5.1) is not correct for processes with a light particle in the final state, for which a factor of $1 \pm f_{p}\left(q_{\text {out }}\right)$ should be added, where the plus sign applies to the case of the Bose distribution (Bose enhancement) an the minus for the Fermi distribution (Pauli blocking). Our calculation differs from the one in $[50,51]$, where the cross section is identified with two times the one for heavy quark-parton scattering computed in [56], supplemented by momentum-independent thermal masses. In our case the Landau damping terms in the EFT approach encode the square of the diagrams for heavy quark-parton scattering, of those for heavy antiquark-parton scattering and the interference term, which depends on the properties of the bound state. We show that in the power counting of the EFT this last term can never be neglected and hence the use of two times the simple heavy quarkparton scattering cross section is inappropriate at weak coupling. Our cross section furthermore does depend on the temperature in all regimes. We plot an example in Fig. 4 for the process $q+\Psi(1 S) \rightarrow q+(Q \bar{Q})_{8}$. The different curves are the cross section computed in different regimes, which show the validity of the employed approximations.

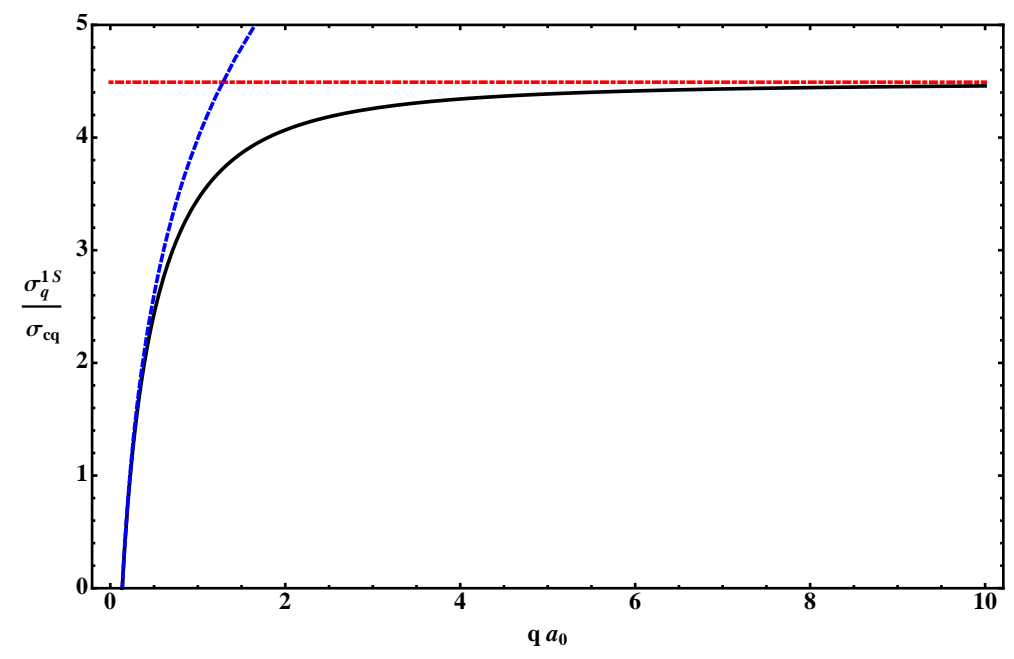

Figure 4: The cross section for $q+\Psi(1 S) \rightarrow q+(Q \bar{Q})_{8}[36,59]$, normalized by the constant $\sigma_{c q} \equiv$ $32 \pi n_{f} \alpha_{\mathrm{s}}^{2} a_{0}^{2} / 3$, where $a_{0}=3 /\left(2 m \alpha_{\mathrm{s}}\right)$ is the Bohr radius, with $m_{D} a_{0}=0.1$. The horizontal axis is the momentum of the incoming quark in units of $a_{0}$. The continuous black line is the cross section for $T \sim m v$, the dashed blue line is the result for $m v \gg T, m_{D} \gg m v^{2}$, obtained in the multipole expansion, and the dashdotted red line is the result for $T \gg m v \gg m_{D}$. The last two curves describe the limiting cases for the first one, which smoothly interpolates between them. We recall that typical thermal constituents of the plasma have momenta of order $T$ and hence $T \sim m v$ translates into $q a_{0} \sim 1$, whereas the blue curve is to be trusted for $q a_{0} \ll 1$ and the red one for $q a_{0} \gg 1$.

\section{Euclidean quantities}

As remarked in the introduction, potential models have often used thermodynamical free energies measured on the lattice as input. Popular choices have been the gauge-invariant colour-average free-energy, derived from the correlator of two Polyakov loops [9], and the colour-singlet free energy [10], which is gauge dependent. 
In [26] the correlator of two Polyakov loops has been analyzed in detail. A short-distance perturbative calculation was carried out to NNLO. Euclidean PNRQCD was then employed to show how the correlator can be expressed in terms of gauge-invariant colour-singlet and colouroctet free energies, which have been computed at short distances. An important finding is that these free energies are not equal to the real-time potentials computed in the same temperature regime: not only are the free energies purely real, in contrast with the complex potentials, but the real parts of the potentials are themselves different from the free energies. The discrepancy has been traced back to the different boundary conditions in the two cases: for the potentials one takes a large Minkowskian time to obtain the pole of the bound-state propagator, whereas for the free energy one has the Euclidean time spanning the entire compactified axis.

The effect of periodic boundary conditions on another gauge-invariant quantity has been analyzed in detail in [31] (see also [63]). There, the renormalization of cyclic Wilson loop, i.e. a rectangular Wilson loop spanning the entire Euclidean time axis, has been studied. This operator had been found to be UV divergent after charge renormalization in [64]. The analysis of [31] shows how this extra divergence is due to the periodic boundary conditions, and how its renormalization involves a mixing with the Polyakov loop correlator. In particular, the difference $W_{c}-P_{c}, W_{c}$ being the cyclic loop and $P_{c}$ the correlator of Polyakov loops, is multiplicatively renormalizable and could then be another interesting quantity to be measured on the lattice and compared with perturbative result, in order to test the applicability of perturbation theory to $Q \bar{Q}$-related quantities.

\section{Non-static and non-perturbative aspects}

The calculations reported in sections 3 and 4 are for a bound state at rest in the medium frame. In [28] (see also [59]) the NR EFT approach was extended to finite relative velocities between the medium and the (abelian) bound state. All the available velocity range, from $v \gtrsim 0$ to $v \rightarrow 1$, was explored. For muonic hydrogen, which is the abelian bound state more closely resembling quarkonium in a medium, it was found that, in the ultrarelativistic limit and for $T>$ $m v$, the imaginary part due to Landau damping loses its role of main dissociation agent in favour of colour screening, whereas for smaller velocities, $0<v<0.9$, the bound state is more easily dissociated for increasing velocities.

The effect of a finite relative velocity was also explored in lattice NRQCD in [65] (see also [47]) for bottomonium $S$-wave states. A momentum dependence of the Euclidean correlators and the reconstructed spectral functions was observed and compared to the EFT results.

A second topic of interest and focus of recent research is the non-perturbative determination of the potential. In $[66,67]$ the spectral function of the Wilson loop has been determined by applying the Maximum Entropy Method to lattice data and a complex potential has then been extracted from it. A different method has been used in $[68,69]$, where a real quantity has been fit from the exponential decay of correlators of temporal Wilson lines of varying time extent at a fixed spatial distance. The strong-coupling version of $\operatorname{pRNQCD}[33,70]$ could be generalized to finite temperatures along the way the weakly-coupled one has; this would help shed more light on how the proper in-medium potential is to be non-perturbatively determined. 


\section{Summary and conclusions}

In this contribution we have briefly illustrated the development and a few chosen results of the NR EFT framework for in-medium quarkonium bound states. From the more theoretical point of view, we have seen how with EFTs one can give a rigorous QCD definition and derivation of the potential, bridging the gap with potentials models which appear as leading-order picture in pNRQCD and its finite-temperature analogues. Furthermore, the power counting EFTs naturally possess allow one to systematically take into account corrections, be it relativistic $1 / \mathrm{m}^{n}$ effects $(n \geq 1)$, radiative corrections, etc. and include all medium effects which are relevant for the desired accuracy.

For what concerns phenomenology, we have shown how previous results, such as the complex potential of [13], are accommodated in the EFT and how new results are obtained [23], such as the estimation of the $\Upsilon(1 S)$ dissociation temperature [27] given in Sec. 3 or the calculation of the corrections to the potential, the spectrum and the width for a Coulombic bound state in the regime $m v \gg T \gg m v^{2}$ [25], illustrated in Sec. 4, which could be of relevance for the new frontier of $\Upsilon$ phenomenology in LHC experiments. We have also shown how the thermal width that arises in the EFT calculations compares with those obtained in phenomenological approaches based on the convolution of $T=0$ cross sections. For what concerns gluo-dissociation, the factorization formula holds and the cross section [55] is a large- $N_{c}$ limit of the EFT cross section [30]. For quasi-free dissociation, instead, the factorization formula employed in the literature is not correct and a direct comparison between the cross sections is not possible [36]. In both cases the power counting allows to constrain the validity region of these approaches.

The EFT framework allows also an analysis of the thermodynamical free energies extracted from correlators of Polyakov loops and widely employed as input for potential models. It is found that the colour-singlet and colour-octet free energies that can be defined in the Euclidean EFT framework differ from the real part of the Minkowski-time potentials, the difference being traced back to the different boundary conditions in the two cases [26], as summarized in Sec. 6. There it is also shown how periodic boundary condition can induce UV divergences in these Euclidean operators, which then mix under renormalization [31].

Possible extensions of the framework include its generalization to the strong-coupling regime, which could help determining which Euclidean operators, amenable to lattice measurements, can be used to extract, possibly through analytical continuation, the real-time potentials governing the evolution of the bound state.

\section{Acknowledgments}

I thank Matthias Berwein, Nora Brambilla, Miguel Ángel Escobedo, Péter Petreczky, Joan Soto and Antonio Vairo for collaboration. I acknowledge financial support from the National Science and Engineering Research Council of Canada and from an Institute of Particle Physics Theory Fellowship.

\section{References}

[1] P. Petreczky, J. Phys. G 39 (2012) 093002 [arXiv:1203.5320 [hep-lat]]. 
[2] T. Matsui and H. Satz, Phys. Lett. B 178 (1986) 416.

[3] N. Brambilla et al., Eur. Phys. J. C 71 (2011) 1534 [arXiv:1010.5827 [hep-ph]].

[4] S. Chatrchyan et al. [CMS Collaboration], arXiv:1208.2826 [nucl-ex].

[5] F. Karsch, M. T. Mehr and H. Satz, Z. Phys. C 37 (1988) 617.

[6] R. Rapp, D. Blaschke and P. Crochet, Prog. Part. Nucl. Phys. 65 (2010) 209 [arXiv:0807.2470 [hep-ph]].

[7] L. Kluberg and H. Satz, arXiv:0901.3831 [hep-ph].

[8] A. Bazavov, P. Petreczky and A. Velytsky, arXiv:0904.1748 [hep-ph].

[9] L. D. McLerran and B. Svetitsky, Phys. Rev. D 24, 450 (1981).

[10] S. Nadkarni, Phys. Rev. D 34 (1986) 3904.

[11] S. Nadkarni, Phys. Rev. D 33 (1986) 3738.

[12] O. Philipsen, Nucl. Phys. A 820 (2009) 33C [arXiv:0810.4685 [hep-ph]].

[13] M. Laine, O. Philipsen, P. Romatschke and M. Tassler, JHEP 0703 (2007) 054 [arXiv:hep-ph/0611300].

[14] A. Beraudo, J. -P. Blaizot and C. Ratti, Nucl. Phys. A 806 (2008) 312 [arXiv:0712.4394 [nucl-th]].

[15] M. Laine, JHEP 0705, 028 (2007) [arXiv:0704.1720 [hep-ph]].

[16] Y. Burnier, M. Laine and M. Vepsalainen, JHEP 0801 (2008) 043 [arXiv:0711.1743 [hep-ph]].

[17] P. Petreczky, C. Miao and A. Mocsy, Nucl. Phys. A 855 (2011) 125 [arXiv:1012.4433 [hep-ph]].

[18] Y. Burnier, M. Laine and M. Vepsalainen, Phys. Lett. B 678 (2009) 86 [arXiv:0903.3467 [hep-ph]].

[19] A. Dumitru, Y. Guo and M. Strickland, Phys. Rev. D 79 (2009) 114003 [arXiv:0903.4703 [hep-ph]].

[20] O. Philipsen and M. Tassler, arXiv:0908.1746 [hep-ph].

[21] M. Strickland, Phys. Rev. Lett. 107 (2011) 132301 [arXiv:1106.2571 [hep-ph]].

[22] M. Strickland and D. Bazow, Nucl. Phys. A 879 (2012) 25 [arXiv:1112.2761 [nucl-th]].

[23] N. Brambilla, J. Ghiglieri, A. Vairo and P. Petreczky, Phys. Rev. D 78 (2008) 014017 [arXiv:0804.0993 [hep-ph]].

[24] M. A. Escobedo and J. Soto, Phys. Rev. A 78 (2008) 032520 [arXiv:0804.0691 [hep-ph]].

[25] N. Brambilla, M. A. Escobedo, J. Ghiglieri, J. Soto and A. Vairo, JHEP 1009 (2010) 038 [arXiv:1007.4156 [hep-ph]].

[26] N. Brambilla, J. Ghiglieri, P. Petreczky and A. Vairo, Phys. Rev. D 82 (2010) 074019 [arXiv:1007.5172 [hep-ph]].

[27] M. A. Escobedo and J. Soto, Phys. Rev. A 82 (2010) 042506 [arXiv:1008.0254 [hep-ph]].

[28] M. A. Escobedo, J. Soto and M. Mannarelli, Phys. Rev. D 84 (2011) 016008 [arXiv:1105.1249 [hep-ph]].

[29] N. Brambilla, M. A. Escobedo, J. Ghiglieri and A. Vairo, JHEP 1107 (2011) 096 [arXiv:1105.4807 [hep-ph]]. 
[30] N. Brambilla, M. A. Escobedo, J. Ghiglieri and A. Vairo, JHEP 1112 (2011) 116 [arXiv:1109.5826 [hep-ph]].

[31] M. Berwein, N. Brambilla, J. Ghiglieri and A. Vairo, arXiv:1212.4413 [hep-th].

[32] A. Pineda and J. Soto, Nucl. Phys. Proc. Suppl. 64 (1998) 428 [arXiv:hep-ph/9707481].

[33] N. Brambilla, A. Pineda, J. Soto and A. Vairo, Nucl. Phys. B 566 (2000) 275 [arXiv:hep-ph/9907240].

[34] W. E. Caswell and G. P. Lepage, Phys. Lett. B 167 (1986) 437.

[35] G. T. Bodwin, E. Braaten and G. P. Lepage, Phys. Rev. D 51 (1995) 1125 [Erratum-ibid. D 55 (1997) 5853].

[36] N. Brambilla, M. A. Escobedo, J. Ghiglieri and A. Vairo, TUM-EFT 27/11, in preparation.

[37] E. Braaten and R. D. Pisarski, Phys. Rev. D 45 (1992) 1827.

[38] A. Vairo, arXiv:1109.2444 [hep-ph].

[39] A. Vairo, PoS CONFINEMENT 8 (2008) 002 [arXiv:0901.3495 [hep-ph]].

[40] J. Ghiglieri, PhD Thesis, TU Munich, 2011, arXiv:1201.2920 [hep-ph].

[41] M. Laine, Nucl. Phys. A 820 (2009) 25C [arXiv:0810.1112 [hep-ph]].

[42] A. Vairo, AIP Conf. Proc. 1317 (2011) 241 [arXiv:1009.6137 [hep-ph]].

[43] B. A. Kniehl and A. A. Penin, Nucl. Phys. B 563 (1999) 200 [hep-ph/9907489].

[44] B. A. Kniehl, A. A. Penin, V. A. Smirnov and M. Steinhauser, Nucl. Phys. B 635 (2002) 357 [hep-ph/0203166].

[45] G. Aarts, S. Kim, M. P. Lombardo, M. B. Oktay, S. M. Ryan, D. K. Sinclair and J. -I. Skullerud, Phys. Rev. Lett. 106 (2011) 061602 [arXiv:1010.3725 [hep-lat]].

[46] G. Aarts, C. Allton, S. Kim, M. P. Lombardo, M. B. Oktay, S. M. Ryan, D. K. Sinclair and J. I. Skullerud, JHEP 1111 (2011) 103 [arXiv:1109.4496 [hep-lat]].

[47] S. M. Ryan, these proceedings.

[48] D. Kharzeev and H. Satz, Phys. Lett. B 334 (1994) 155 [arXiv:hep-ph/9405414].

[49] X. M. Xu, D. Kharzeev, H. Satz and X. N. Wang, Phys. Rev. C 53 (1996) 3051 [arXiv:hep-ph/9511331].

[50] L. Grandchamp and R. Rapp, Phys. Lett. B 523 (2001) 60 [hep-ph/0103124].

[51] L. Grandchamp and R. Rapp, Nucl. Phys. A 709 (2002) 415 [hep-ph/0205305].

[52] X. Zhao and R. Rapp, Phys. Rev. C 82 (2010) 064905 [arXiv:1008.5328 [hep-ph]].

[53] A. Emerick, X. Zhao and R. Rapp, Eur. Phys. J. A 48 (2012) 72 [arXiv:1111.6537 [hep-ph]].

[54] T. Song, K. C. Han and C. M. Ko, Phys. Rev. C 85 (2012) 014902 [arXiv:1112.0613 [nucl-th]].

[55] G. Bhanot and M. E. Peskin, Nucl. Phys. B 156 (1979) 391.

[56] B. L. Combridge, Nucl. Phys. B 151 (1979) 429.

[57] T. Song and S. H. Lee, Phys. Rev. D 72 (2005) 034002 [hep-ph/0501252].

[58] Y. Park, K. I. Kim, T. Song, S. H. Lee and C. Y. Wong, Phys. Rev. C 76 (2007) 044907 [arXiv:0704.3770 [hep-ph]]. 
[59] M. A. Escobedo, these proceedings, TUM-EFT 37/13.

[60] F. Brezinski and G. Wolschin, Phys. Lett. B 707 (2012) 534 [arXiv:1109.0211 [hep-ph]].

[61] F. Nendzig and G. Wolschin, arXiv:1210.8366 [hep-ph].

[62] G. Wolschin, these proceedings.

[63] M. Berwein, these proceedings, TUM-EFT 39/13.

[64] Y. Burnier, M. Laine and M. Vepsalainen, JHEP 1001 (2010) 054 [arXiv:0911.3480 [hep-ph]].

[65] G. Aarts, C. Allton, S. Kim, M. P. Lombardo, M. B. Oktay, S. M. Ryan, D. K. Sinclair and J. -I. Skullerud, arXiv:1210.2903 [hep-lat].

[66] A. Rothkopf, T. Hatsuda and S. Sasaki, Phys. Rev. Lett. 108 (2012) 162001 [arXiv:1108.1579 [hep-lat]].

[67] Y. Burnier and A. Rothkopf, Phys. Rev. D 86 (2012) 051503 [arXiv:1208.1899 [hep-ph]].

[68] A. Bazavov and P. Petreczky, arXiv:1210.6314 [hep-lat].

[69] A. Bazavov and P. Petreczky, arXiv:1211.5638 [hep-lat].

[70] N. Brambilla, A. Pineda, J. Soto and A. Vairo, Rev. Mod. Phys. 77 (2005) 1423 [hep-ph/0410047]. 